\title{
Blood virosphere in febrile Tanzanian children
}

\begin{abstract}
Samuel Cordey (1D ${ }^{a, b *}$, Florian Laubscher ${ }^{a, b_{*}}$, Mary-Anne Hartley ${ }^{c, d_{*}}$, Thomas Junier ${ }^{\mathrm{e}, \mathrm{f}}$, Kristina Keitel ${ }^{\mathrm{g}, \mathrm{h}}$,

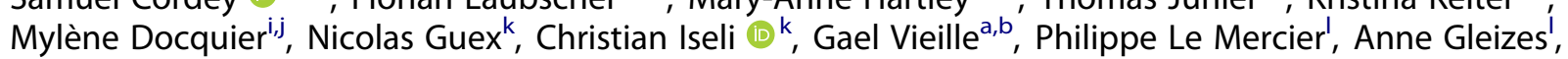
Josephine Samaka ${ }^{m}$, Tarsis Mlaganile ${ }^{m}$, Frank Kagoro ${ }^{m}$, John Masimba ${ }^{m}$, Zamzam Said ${ }^{m}$, Hosiana Temba ${ }^{m}$, Gasser H. Elbanna ${ }^{d}$, Caroline Tapparel ${ }^{\mathrm{n}}$, Marie-Celine Zanella ${ }^{\mathrm{a}, \mathrm{b}}$, Ioannis Xenarios ${ }^{\mathrm{o}, \mathrm{p}}$, Jacques Fellay ${ }^{\mathrm{e}, \mathrm{f}, \mathrm{q}}$, Valérie $D^{\prime}$ Acremont ${ }^{c, g}+$ and Laurent Kaiser ${ }^{\mathrm{a}, \mathrm{b}, \mathrm{r}}+$

${ }^{a}$ Division of Infectious Diseases, Geneva University Hospitals, Geneva, Switzerland; ${ }^{\mathrm{b}}$ Laboratory of Virology, Division of Infectious Diseases and Division of Laboratory Medicine, University Hospitals of Geneva \& Faculty of Medicine, University of Geneva, Geneva, Switzerland; 'Centre for Primary Care and Public Health (Unisanté), University of Lausanne, Lausanne, Switzerland; Intelligent Global Health, Machine Learning and Optimization Laboratory, EPFL, Lausanne, Switzerland; ${ }^{\mathrm{E}} \mathrm{Global}$ Health Institute, School of Life Sciences, EPFL, Lausanne, Switzerland; 'SIB Swiss Institute of Bioinformatics, Lausanne, Switzerland; 'S Swiss Tropical and Public Health Institute, University of Basel, Basel, Switzerland; ' Department of Paediatric Emergency Medicine, Department of Pediatrics, Inselspital, Bern University Hospital, University of Bern, Bern, Switzerland; i iGE3 Genomics Platform, University of Geneva, Geneva, Switzerland; 'Department of Genetics and Evolution, University of Geneva, Geneva, Switzerland.; 'Bioinformatics Competence Center, University of Lausanne and EPFL, Lausanne,

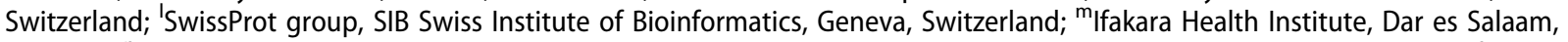
Tanzania; ${ }^{n}$ Department of Microbiology and Molecular Medicine, University of Geneva Medical School, Geneva, Switzerland; ${ }^{\circ} \mathrm{Health} 2030$ Genome Center, Geneva, Switzerland; ${ }^{\mathrm{p}}$ Agora Center, University of Lausanne, Lausanne, Switzerland; ${ }^{9}$ Precision Medicine Unit, Lausanne University Hospital and University of Lausanne, Lausanne, Switzerland; 'Geneva Centre for Emerging Viral Diseases, Geneva University Hospitals, Geneva, Switzerland
\end{abstract}

\section{ABSTRACT}

Viral infections are the leading cause of childhood acute febrile illnesses motivating consultation in sub-Saharan Africa. The majority of causal viruses are never identified in low-resource clinical settings as such testing is either not part of routine screening or available diagnostic tools have limited ability to detect new/unexpected viral variants. An indepth exploration of the blood virome is therefore necessary to clarify the potential viral origin of fever in children. Metagenomic next-generation sequencing is a powerful tool for such broad investigations, allowing the detection of RNA and DNA viral genomes. Here, we describe the blood virome of 816 febrile children ( $<5$ years) presenting at outpatient departments in Dar es Salaam over one-year. We show that half of the patients (394/816) had at least one detected virus recognized as causes of human infection/disease $(13.8 \%$ enteroviruses (enterovirus $A, B, C$, and rhinovirus $A$ and $C), 12 \%$ rotaviruses, $11 \%$ human herpesvirus type 6 ). Additionally, we report the detection of a large number of viruses (related to arthropod, vertebrate or mammalian viral species) not yet known to cause human infection/disease, highlighting those who should be on the radar, deserve specific attention in the febrile paediatric population and, more broadly, for surveillance of emerging pathogens.

Trial registration: ClinicalTrials.gov identifier: NCT02225769.

ARTICLE HISTORY Received 15 February 2021; Revised 13 April 2021; Accepted 28 April 2021

KEYWORDS Blood virome; virosphere; metagenomic next-generation sequencing; fever; children

\section{Introduction}

Humans are infected by a large number of eukaryotic DNA and RNA viruses that, together with prokaryotic phages and endogenous viral elements, comprise what we define as the human virome. Eukaryotic viruses are a diverse group of DNA and RNA viral agents replicating in a variety of human cells and tissues and engaging in a vast permutation of interactions with the human immune system. Consequently, the presentation of human viral disease depends on both viral and host factors, where a virus may only be pathological in a certain situation (e.g. following immunosuppression), whilst being considered (perhaps over simplistically) as commensal or merely an incidental finding in a "healthy" situation.

The composition of the human virome does not only vary between these populations but it also changes over time within individuals according to their personal history of exposure and immunity and thus, their geographic location, access to vaccination

CONTACT Samuel Cordey samuel.cordey@hcuge.ch E Laboratory of Virology, University of Geneva Hospitals, 4 rue Gabrielle-Perret-Gentil, Geneva 1205, Switzerland

*Equal contribution.

${ }^{\dagger}$ These senior authors contributed equally to this article.

(4) Supplemental data for this article can be accessed at https://doi.org/10.1080/22221751.2021.1925161.

(c) 2021 The Author(s). Published by Informa UK Limited, trading as Taylor \& Francis Group.

This is an Open Access article distributed under the terms of the Creative Commons Attribution License (http://creativecommons.org/licenses/by/4.0/), which permits unrest ricted use, distribution, and reproduction in any medium, provided the original work is properly cited. 
and their genetic make-up. Metagenomic next-generation sequencing ( $\mathrm{mNGS}$ ) has the ability to detect any viral RNA or DNA sequence in a given sample and thus, it represents the most appropriate tool with which to explore the human virome [1].

It is recognized that we have only uncovered a fraction of the so-called virosphere $[2,3]$. While many human viruses have emerged from animal origin, shotgun surveillance in sentinel animals generates a lot of noise by reporting a majority of viruses unlikely to infect humans. Thus, we hypothesize that pathogen surveillance would be extremely informative to public health interventions if focused first on humans. Specifically, there is a need for investigations that could reveal not only the presence of known and expected viruses, but also divergent or unexpected ones. Characterization of the human virome could help to identify new sources of infections, possibly predict viral emergence or identify the potential deleterious effects of viruses considered as commensal.

The human virome has been described in stool, skin, nasopharyngeal swabs or breast milk [4]. Investigations were also undertaken on blood from individuals representing the general population [5], blood donors [6-9], and in subjects with various conditions such as transplant recipients [10-12], individuals living with HIV [13], and febrile adult and paediatric patients [1, 14-18]. However, most of these studies were limited by the scope of their analyses (e.g. addressing only RNA or DNA viruses, pooled samples) or in the time frame of included subjects.

Infections and fever in sub-Saharan Africa represent the most frequent motivation for paediatric outpatient consultation [19]. The majority of causal viruses, however, are never identified in low-resource clinical settings. In this study, we seek to characterize the blood virome in a cohort of 823 children consulting at nine outpatient departments in Dar es Salaam, Tanzania, for acute fever of unknown origin and/or severe outcomes (Figure 1) [20]. We tested each sample retrospectively by mNGS and analysed them using (a) a bioinformatic pipeline based on a novel viral reference database [21] to report vertebrate viruses and (b) by de novo assembly. We report the full diversity of their blood virome, including novel viruses and those unexpected or not previously reported in humans that deserve specific attention.

\section{Materials and methods}

\section{Study design}

The serum samples tested by mNGS come from a larger cohort of 3192 consecutively paediatric patients (2-59 months of age), recruited between December 2014 and February 2016, at nine outpatient clinics in Dar es Salaam, Tanzania, with the inclusion criteria of "acute febrile illness" (axillary temperature $\geq 37.5^{\circ}$ C). The detailed characteristics of this cohort are described in Keitel et al [20].

A sub-cohort of interest comprising 1233 patients was then selected (Figure 1) based on three diagnoses which carried the highest theoretical probability of detecting blood viruses with significant clinical consequences and for which mNGS would most increase the diagnostic evidence for the aetiology of the illness. These diagnoses were obtained using by the clinical decision algorithms of the e-POCT study [20]: (1) "Fever without focus" representing children who would most benefit from further diagnostics; (2) "Severe illness," representing children in whom the impact of blood viruses may be investigated. (3) "Malaria," in whom blood viruses may interact with this important and frequent parasite and/or be less likely as the cause of the febrile episode.

A total of 823 patients (resulting in 823 sera samples) were then randomized from this sub-cohort of interest and submitted retrospectively to the entire process of mNGS (detailed below). Only samples with a minimum of one million reads for both RNA and DNA libraries were considered for further bioinformatic analysis, resulting in the exclusion of 4 samples. Three samples were further excluded; two consecutive to a technical problem encountered during the library procedures, and one due to an overrepresentation of insect sequences after analysis of raw sequencing data [22]. Therefore, 816 of the 823 initial selected samples were considered for analysis.

\section{Ethical approval}

This study was approved by the local IRB in Tanzania by the Ifakara Health Institute and the National Institute for Medical research (paediatric cohort: IHI/IRB/ EXT/16-2015 and NIMR/HQ/R.8a/Vol. IX/1789). IRB approval was also granted for analyses undertaken in Switzerland by the Ethikkommission Nordwestund Zentralschweiz (EKNZ UBE-15/03). Written informed consent was obtained from the parent or guardian of the patients before enrolment. The trial was registered in ClinicalTrials.gov, identifier NCT02225769.

\section{Sample extraction and metagenomic next- generation sequencing}

Two hundred twenty microliters (ul) of each serum sample was centrifuged $10,000 \times g$ for $10 \mathrm{~min}$. Then, $200 \mathrm{ul}$ are transferred in a new collection tube and treated with $40 \mathrm{U}$ of Turbo DNAse $(2 \mathrm{U} / \mathrm{ul})+24 \mathrm{ul}$ of 10X DNAse Buffer (Ambion, Rotkreuz, Switzerland). Of the total resulting 244, $240 \mu$ l were recovered to perform the nucleic acid extraction. Two nucleic acid extraction procedures were then carried out 


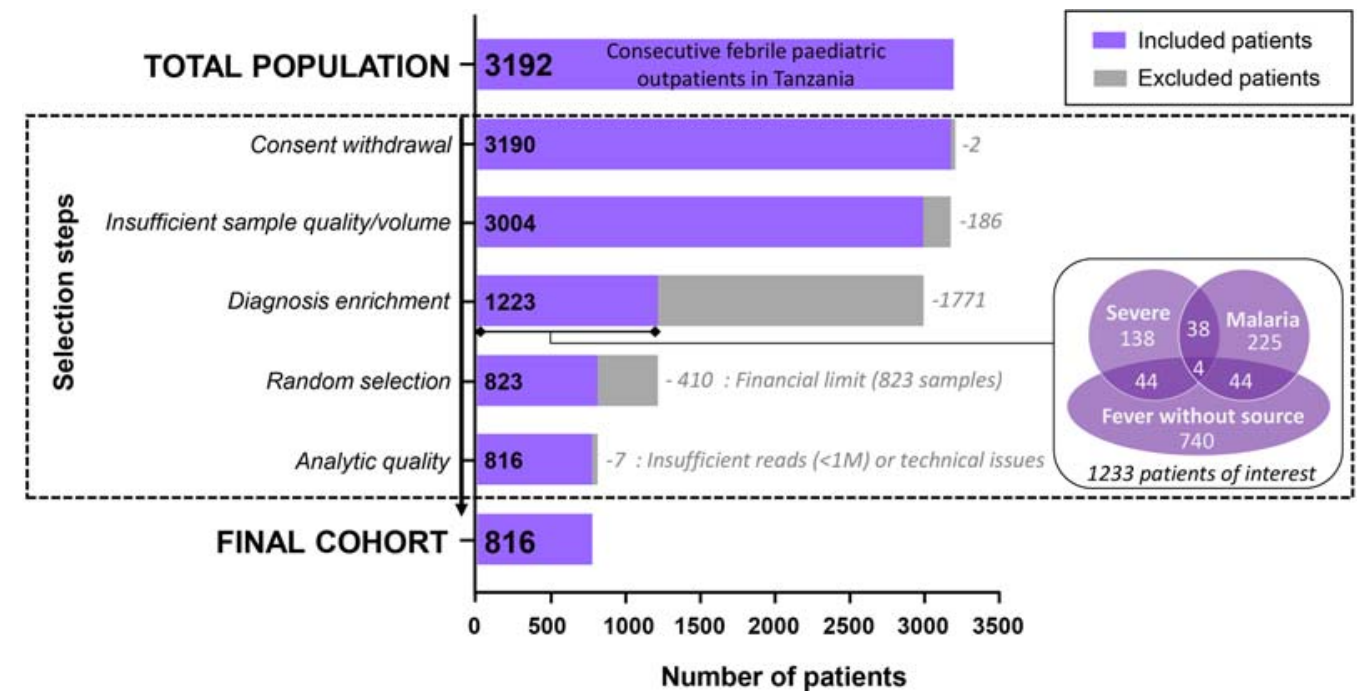

Figure 1. Flowchart of study selection.

separately for RNA and DNA virus genome extraction (i.e. RNA and DNA protocols, respectively) as previously described [23]. Briefly, RNA genome extraction (RNA protocol) was isolated with TRIzol (Invitrogen, Carlsbad, CA, USA), taking care to collect only the upper aqueous layer to avoid DNA contaminations from the inter-/lower phases. DNA virus genome extraction (DNA protocol) was performed with the NucliSens easyMAG magnetic bead system (bioMérieux, Geneva, Switzerland) followed by a doublestranded DNA synthesis with DNA polymerase I, Large Fragment (Klenow) (New England BioLabs, Ipswich, MA, USA). RNA and DNA pellets were then resuspended in respectively 10 and $5 \mathrm{ul}$ of nucleasefree water (Promega, Dübendorf, Switzerland), and quantified using the Qubit 3.0 Fluorometer (Life Technologies, Carlsbad, CA, USA) and adjusted at $2 \mathrm{ng} / \mathrm{ul}$.

For RNA virus detection (RNA protocol), ribosomal RNA was removed using the Ribo-Zero Gold depletion kit (Illumina, San Diego, US) prior to library preparation. RNA libraries were prepared with the TruSeq total RNA preparation protocol (Illumina) before being multiplexed by four on the HiSeq 2500 platform (Illumina) using the $2 \times 100 \mathrm{bp}$ paired-end protocol. The mean of total number of read pairs obtained was 4.45E7 (range from 2E6 to 1.09E8).

For DNA virus detection (DNA protocol), libraries were prepared with the Illumina Nextera XT protocol (12 PCR cycles) before being multiplexed by six on the HiSeq 4000 platform (Illumina) using the $2 \times 100 \mathrm{bp}$ paired-end protocol. The mean of total number of read pairs obtained was 5.46E7 (range from 2E6 to 2.52E8).

Both RNA and DNA library concentrations were measured using Qubit (Life Technologies). The size distribution of fragments was controlled using a 2200 TapeStation (Agilent, Santa Clara, CA, USA).

To assess the presence of potential mNGS contaminants (i.e. false positive results), a "no-template" control (NTC) submitted to the whole procedure was included to each sequencing run. In order to assess the entire process efficiency (i.e. from sample preparation to bioinformatics analysis) each sequencing run included a virus-spiked positive control (PC). This latter is also useful in addition to the NTC samples to evaluate the presence of potential sporadic mNGS contaminants. For the RNA virus procedure, the PC consists of a canine distemper virus (CDV)-spiked positive control (RNA-PC), whereas for the DNA virus procedure, a baculovirus (GenScript, Piscataway, NJ, USA) harboring 793 nucleotides of the CDV fusion gene (corresponding to positions 5505 to 6297 from the GenBank KY971529 reference) was used as spiked-positive controls (DNA-PC).

\section{Bioinformatic analysis}

For each sample, the reads generated by the Illumina platforms (RNA and DNA libraries) were processed for virus detection using a bioinformatics pipeline (FeVir) designed to detect all vertebrate viruses and a de novo assembly sequencing approach.

\section{Bioinformatics pipeline for vertebrate virus detection (FeVir)}

Paired reads were quality filtered and mapped against Virosaurus (version V90v_2018_11) [21], a curated database for all known vertebrate viruses, using virusscan 1.0 [24]. The mapping was performed allowing up to 12 mismatches per tag but at most 15 mismatches for the pair, ultimately retaining the lowest possible mismatch. Sequence pairs whose lowest mismatch count mapped equally well onto more than 512 distinct viruses entries were discarded. Non-redundant mapping results were used to compute metrics for 
mapped reads and coverage. Then for each virus species (or genus for anelloviridae) detected, results with the best total genome coverage value were kept. To minimize false positives, only results with coverage $\geq 300$ nucleotides (i.e. a minimal of 3 independent and non-overlapping reads) were considered as positive $[23,25]$.

Index hopping/lane cross-contamination was removed using a $1 \%$ threshold. An important challenge in the interpretation of mNGS data is to distinguish clinically significant viruses from those that are known to be medically irrelevant and also to differentiate novel viruses (not previously described in humans) from those that are more likely to be environmental or mNGS reagent contaminants [26]. Therefore, results for viruses detected in processes controls and endogenous retrovirus were not reported.

For segmented viruses, results for each segment were summed. For Herpesviridae, each gene's results were summed using a non-redundant and non-overlapping list of genes.

Viral hits were classified into 3 groups:

(1) Those of "recognized clinical significance" including viruses known to infect humans and well recognized in clinical practice as disease-causing agents. As for most pathogens the spectrum of associated illness associated with this group of viruses ranges from mild symptoms (or even asymptomatic infection) to life-threatening disease.

(2) Commensal viruses of "undetermined clinical significance" that comprise viruses that infect human but for which the association with a specific disease has not yet been established. Most of these viruses however have shown to interact with the human immune response and have been referred to by some as commensal agents. The two main representatives are Anelloviridae and human pegivirus-1.

(3) Those of "unknown significance" that represent viral agents not usually recognized as infecting humans and/or have never fulfilled Koch's postulates that would attribute a theorical pathogenic role [27]. Thus, it could reflect transient or aborted infections, environmental spill over, contaminants from skin or other body fluids or spurious mNGS reagent contaminants. We consider this group of particular interest to surveillance efforts, since it could signal contact with potential emerging human pathogens and guide targeted screening in the future.

\section{De novo analysis}

The de novo analysis was performed as previously published [28]. First, reads were trimmed to remove low-quality and adapter sequences using Trimmomatic (v0.33). Human reads were removed by mapping reads against the human genome and transcriptome (hg38, gencode.V23) using the SNAP nucleotide aligner program [29]. Then, de novo assembly was performed using IDBA-UD (v.1.1.3) [30] and the generated contigs of greater than 2000 nucleotides were blasted (blastx, v.2.3.0+) [31] against the URVDBv12.2 viral database [32]. Anelloviridae contigs were filtered out and processed separately. The newly obtained sequences were then cross-checked by BLAST (blastn, blastx) against GenBank (Nucleotid collection $(\mathrm{nr} / \mathrm{nt})$, Non-redundant protein sequences $(\mathrm{nr})$ ) and NCBI conserved domain tool. Novel virus contigs with a presumed bacterial or fungi host were not considered for further analyses.

Sample and control FASTQ files were additionally filtered for low-complexity sequences, using tagdust (v2.31). Remaining reads were mapped against assembled genomes that were not already detected by the previously described FeVir pipeline, using SNAP. For each new hit, mapped reads and coverage metrics were then computed. Only results with $\geq 300$ nucleotides of coverage were considered. Results for viruses detected in processes controls were not reported.

\section{Anelloviridae sequence analysis}

Anelloviridae contigs with a complete ORF1 larger than 600 amino acids were separated into Alpha-, Beta- and Gamma-torquevirus genera using multiple alignment (muscle v3.8.31) with the reference sequences of ORF1. Alternative "ACG" initiation codon was also used to detect Alphatorquevirus ORF1.

To estimate the proportion of assembled contigs that was "new" compared to reported sequences in GenBank (Query: alphatorquevirus [Organism] OR betatorquevirus [Organism] OR gammatorquevirus [Organism] OR unclassified Anelloviridae [Organism], downloaded in March 2019), we first excluded non-primate sequences, then filtered as above and added to the new assembled sequences. For the three genera, complete ORF-1 sequences were separated in clusters of $80 \%$ nucleotide identity, using cdhit-est (CD-HIT v4.6). For each cluster, sequences of $90 \%$ identity were selected using cdhit-est. Then representative sequences were aligned two by two using muscle (v3.8.1551), when two clusters contain reference sequences that shared more than $80 \%$ nucleotide identity, clusters were merged.

To determine the number of distinct Anelloviridae of the same genus present in each sample, the filtered FASTQ files were mapped to the ORF1 representative sequence using SNAP, for each cluster, positive detection was considered if $50 \%$ or more of the ORF 1 was covered. 
Additionally, for each of the assembled Anelloviridae contigs that contained a complete ORF1, circular genomes were linearized starting after the GC rich region and incomplete genomes were trimmed the same way. Finally, representative genomes were selected at $90 \%$ nucleotide identity with cdhit-est. Then filtered FASTQ files were mapped against the representative genomes with SNAP. Metrics were computed and results were processed as described above $(\geq 300$ nucleotides, $1 \%$ index-hopping/lane cross-contamination threshold). Results with the best total genome coverage value for each genus were reported if previously found negative with the FeVir pipeline.

\section{Results}

In this large population of febrile paediatric outpatients recruited in East Africa, we report the detection of a large diversity of RNA and DNA virus sequences that we classify into 3 groups (Supplementary Figure 1, https://www.unige.ch/virology/en/krona/); those known to cause disease in humans and of "recognized clinical significance" (Supplementary Figure 1(a)), those known to be present in humans and of "undetermined clinical significance" (Supplementary Figure 1 (b)) and a third group of viruses not usually described in humans and of "unknown significance" (Supplementary Figure 1(c)).

\section{Viruses of "recognized clinical significance"}

Of the 816 children, 394 (48.3\%) harboured RNA and/ or DNA sequences specific for a virus recognized as causing human diseases (Supplementary Table 1). This group was dominated by RNA viruses, predominantly from members of the Picornaviridae family such as human enteroviruses (EV) $(13.8 \%$ of all cases, 113/816) and rotaviruses (12\% of all cases, 98/ 816) (Supplementary Figure 1(a)).

Species from various genera of the Picornaviridae family were detected, including EV-A, B and C ( $n=$ 39,31 and 1, respectively), rhinovirus (RV)-A and $\mathrm{C}$ ( $n=3$ and 39 , respectively), hepatovirus $\mathrm{A}(n=10)$ and parechovirus $\mathrm{A}(n=9)$ (Figure $2(\mathrm{a}))$. Within the different EV species, various coxsackievirus $A$ and echovirus genotypes were observed (Supplementary Table 1), as well as one EV-A71 case that may represent a new EV-A71 genogroup (Supplementary Figure 2, SAfia-57/TZA/2016 MN727165.1). Among EV-C, poliovirus-related sequences were not observed. A large diversity of RV-C were detected (Supplementary Figure 3), nine of which were only detectable by de novo analysis (Figure 2(b)), highlighting the absence of similar sequences in any available database. The $17 \mathrm{RV}-\mathrm{C}$ for which a complete VP1 sequence was recovered, could be clustered into 9 distinct RV types (including one that may, tentatively, be categorized as a new type). One of the genomes (SAfia94/TZA/2015 MN727266.1) is an RV-C45/RV-C11 recombinant similar to a variant described in a wild chimpanzee in Uganda [33].

A total of $98 / 816(12 \%)$ samples had detectable rotavirus sequences, and only one corresponded to the vaccine G1P [8] strain (Supplementary Figure 1 (a)). Human astroviruses (HAstV) were the $3 \mathrm{rd}$ most frequently detected among RNA viruses from this group. The classical HAstV (i.e. HAstV serotypes 18 ) that are a well-known cause of gastroenteritis, represented only a minority of cases $(n=7)$ whereas the novel HAstV (i.e. HAstV MLB/VA) were observed in 17 cases (4 MLB1, 2 MLB2, 5 VA1 and 6 VA3). We also identified Norwalk virus $(n=12)$, human immunodeficiency virus $1(n=7)$, Sapporo virus $(n$ $=4)$, human coronavirus OC43 $(n=2)$, human parainfluenza virus type $4 \mathrm{a}(n=1)$ and the Wistar RA $27 / 3$ rubella vaccine strain in one case. Finally, only one patient had detectable sequences of dengue virus (serotype 2), which corroborates clinical diagnoses and on-site rapid testing, that the study did not occur during a dengue virus outbreak.

Concerning the DNA viruses, by far the most frequent ones were members of the Betaherpesvirinae subfamily followed by mastadenovirus species. Notably, a significant proportion of these agents were also recovered at the RNA level (i.e. RNA protocol).

Among Betaherpesvirinae (Supplementary Figure 1 (a)), human herpesvirus type 6 (HHV-6) was the most frequent ( $n=90 ; 63$ belonging to the HHV-6 B species and 27 remaining untyped), followed by human herpesvirus type $7(\mathrm{HHV}-7)(n=36)$ and cytomegalovirus $(\mathrm{CMV})(n=15)$ (Figure $2(\mathrm{c}, \mathrm{d}))$. In addition, the following herpesviruses were identified in a few samples: varicella zoster virus $(n=4)$, herpes simplex type 1 virus $(n=1)$, and human herpesvirus $8 \quad(n=1)$. Among all HHV-6, HHV-7 and CMV positive samples listed above, a large proportion (73.3\%, $75.7 \%$ and $33.3 \%$, respectively) were detected in both DNA and RNA libraries suggesting the presence of ongoing genome replication. Of note, $7 \mathrm{HHV}-6,2$ HHV-7 and $5 \mathrm{CMV}$ positive cases were detected in the RNA libraries only, which means that, overall, $81.1 \%$ of HHV $-6(n=73 / 90), 83.3 \%$ of HHV -7 ( $n=$ $30 / 36)$ and $66.6 \%$ of CMV $(n=10 / 15)$ positive cases were detected in the RNA libraries.

Specific sequences for human mastadenovirus (HAdV) were recovered in 28 samples and encompassed species A, C, E and F. Human bocavirus, parvovirus B19, trichodysplasia spinulosa-associated polyomavirus and $\mathrm{BK}$ virus were recovered in 10 or less samples each (Figure 2(c)). A fraction of these DNA viruses was also detected in the RNA libraries only (five HAdVs (one each for species A, E and F, two for the species $\mathrm{C}$ ) and one human bocavirus) (Figure 2(c)). 

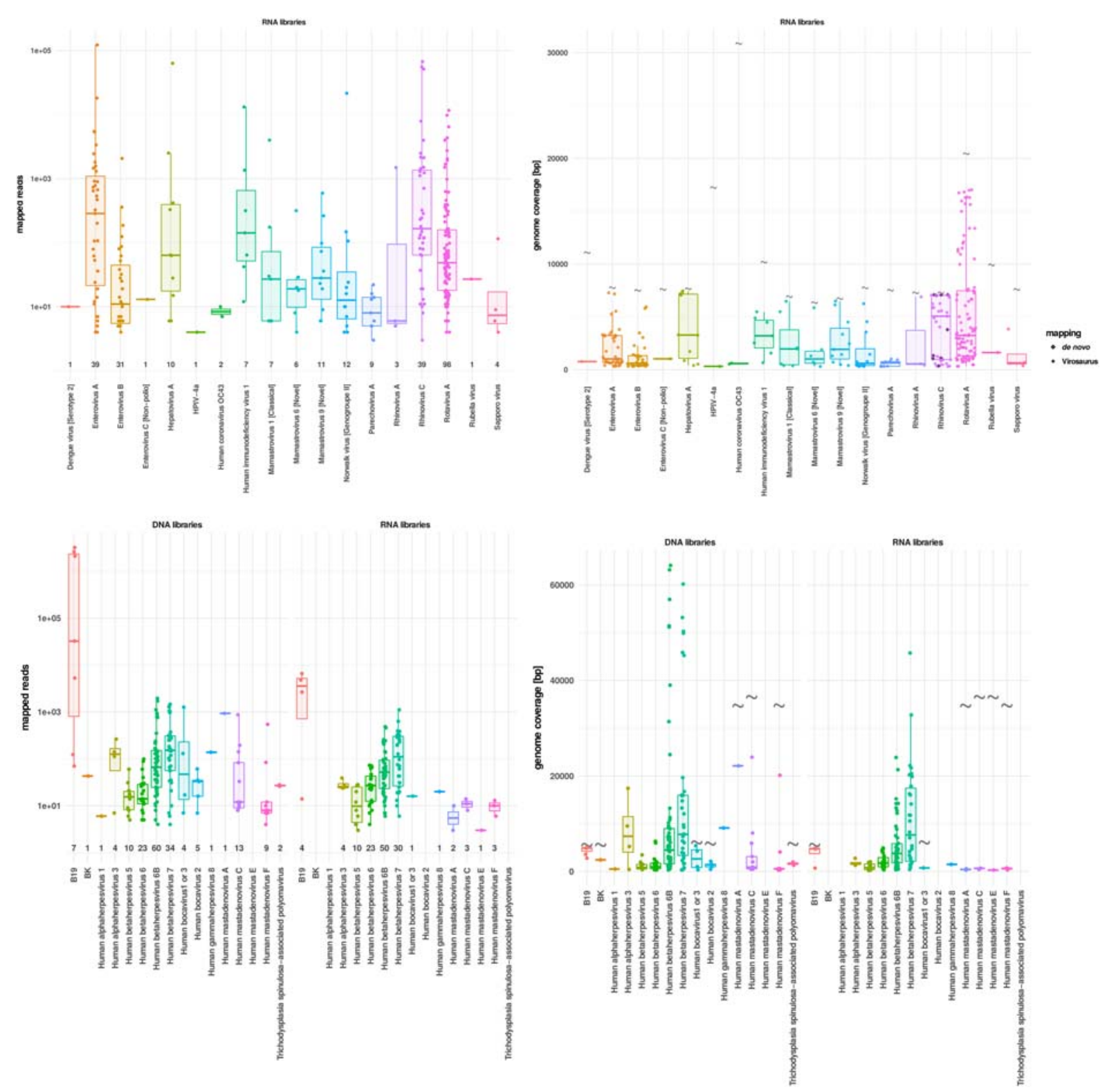

Figure 2. mNGS metrics for viruses of recognized clinical significance. (a) Distribution of the mapped reads for RNA viruses. Each dot represents a positive sample. (b) RNA virus genome coverages. Viral sequences detected by de novo only are represented by grey diamonds. Each dot represents one sample. Viral full genome sizes (bp) are indicated by grey corrugated symbols. (c) Distribution of the mapped reads for DNA viruses detected in DNA (left side) and RNA (right side) libraries. Each dot represents a positive sample. (d) DNA virus genome coverages in DNA (left side) and RNA (right side) libraries. Each dot represents one sample. Viral full genome sizes (bp) are indicated by grey corrugated symbols (except for herpesviruses that have too long genomes to figure in the panel). The horizontal lines in the box plots denote medians.

\section{Commensal viruses of "undetermined clinical significance"}

Anelloviridae sequences were detected in all except one clinical sample (Supplementary Figure 1(b) and Figure 3). Co-infection with multiple genera was the rule $(99.0 \%)$, with $752 / 816$ samples found positive for all 3 genera (Figure 4(a)). Torque teno mini virus (TTMV, $n=803$ ) was the most frequent, followed by Torque teno virus (TTV, $n=795)$, and Torque teno midi virus (TTMDV, $n=776$ ) (Figure $3(\mathrm{c}, \mathrm{d}$ ) and Figure 4(a)). Sequence analyses revealed a very high genetic heterogeneity (Figure 4(b)) with an extremely large number of divergent and/or different genotypes from the same genus present in a single sample
(Figure 4(c)). As an example, up to 69 different TTV, 259 TTMV and 189 TTMDV genotypes were observed in a single clinical sample (Supplementary Table 1, sample ID 25161).

In addition, we detected sequences corresponding to adeno-associated dependoparvovirus $\mathrm{A}(n=69)$, human pegivirus-1 $(\mathrm{HPgV}-1)(n=42)$, cardiovirus $\mathrm{B}$ $(n=2)$, and human polyomavirus $10(n=1)$ (Supplementary Figure 1(b) and Figure $3(\mathrm{a}-\mathrm{d})$ ).

\section{Viruses of "unknown significance"}

A large number of sequences related to arthropod, vertebrate or mammalian viruses (DNA and RNA-based) 


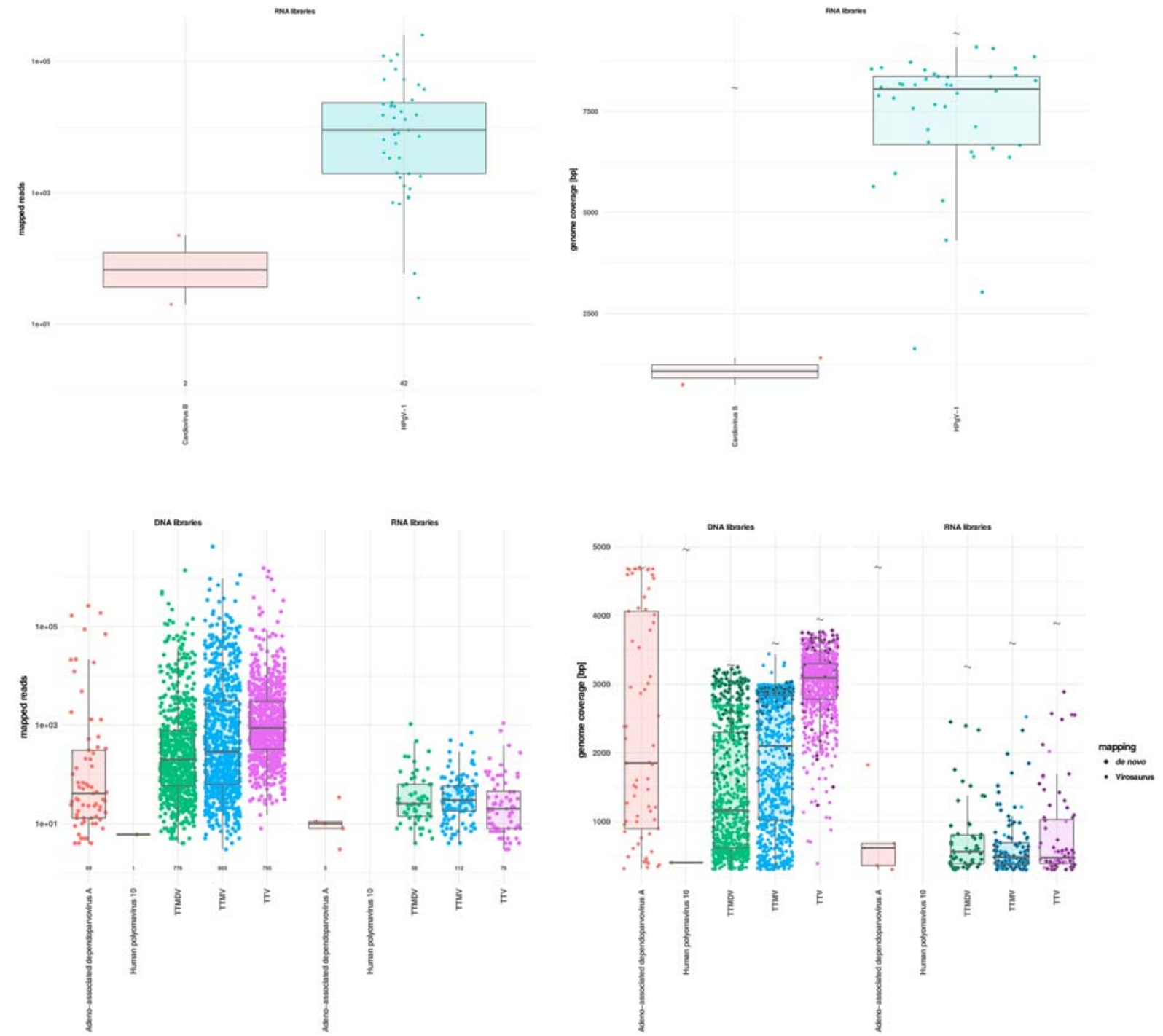

Figure 3. mNGS metrics for viruses of undetermined clinical significance. (a) Distribution of the mapped reads for RNA viruses. Each dot represents a positive sample. (b) RNA virus genome coverages. Each dot represents one sample. Viral full genome sizes (bp) are indicated by grey wave symbols. (c) Distribution of the mapped reads for DNA viruses detected in DNA (left side) and RNA (right side) libraries. Each dot represents a positive sample. (d) DNA virus genome coverages in DNA (left side) and RNA (right side) libraries. Each dot represents one sample. Viral full genome sizes (bp) are indicated by grey corrugated symbols. Viral sequences detected by de novo only are represented by grey diamonds. The horizontal lines in the box plots denote medians.

that are not recognized as a cause of infection in humans were detected using either the FeVir pipeline and/or de novo analysis (Supplementary Figure 1(c) and Figure 5). We describe a tentative novel Dicistroviridae genus (a family of viruses known to infect arthropods) identified in 84 samples and reported separately in Cordey et al [34]. Porcine parvoviruses 4-6 $(n=50)$ were also detected, including two nearly complete sequences (Figure 5(c, d)). Ambidensoviruses 13 , known to infect insects and other non-vertebrates, were present $(n=44)$ with a significant number of reads detected in the DNA libraries (Figure 5(c, d)).

Several additional viruses for which no RNA or DNA sequences were detected in the NTC and PC samples were observed. At the RNA level, several viruses were detected that could be reported as "-like" known viruses, without however matching closely to them. This included sequences for picorna-like virus $(n=27)$, providence-like viruses 1-2 $(n=24)$, dicistro-like viruses 1-2 $(n=12)$, bunya-like virus 1-3 $(n=3)$, and phlebovirus-like virus $(n=1)$ (Figure $5(\mathrm{a}, \mathrm{b}))$. Others were rotavirus $\mathrm{D}(n=2)$, avian coronavirus $(n=1)$, iflavirus $(n=1)$, and mammalian orthoreovirus $(n=1)$ (Figure 5(a, b)). Among DNA viruses, sequences were also detected for human associated gemykibivirus ( $n=$ $29)$, geminivirus $(n=11)$, human associated cycloviruses $(n=5)$, chicken stool-associated gemycircularvirus $(n=2)$, CRESS virus $(n=2)$, cyclovirus ZM36a $(n=2)$, avian gyrovirus $2(n=1$, RNA library only), bat associated cyclovirus $16(n=1)$, human associated gemyvongvirus 1 ( $n=1$, RNA library only), human CSF-associated densovirus $(n=1)$, and taupapillomavirus $3(n=1)$ (Figure 5(c, d)). 


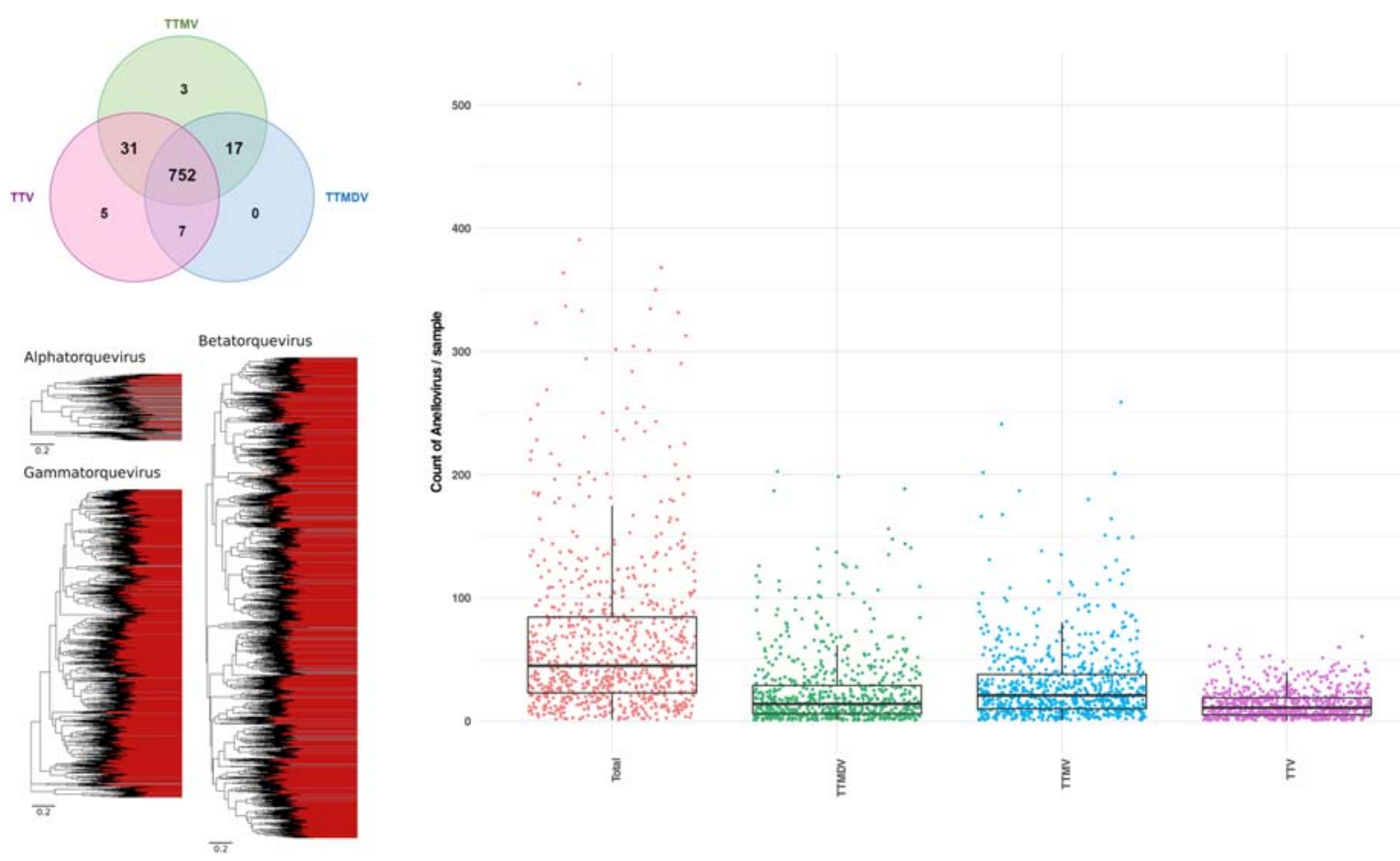

Figure 4. Representation of Anelloviridae co-detection. (a) Venn plot showing the distribution of Alpha-, Beta- and Gammatorquevirus genus among positive samples. (b) Phylogenetic diversity of primate anellovirus. Alpha-, Beta- and Gammatorquevirus genus are represented by three Maximum Likelihood trees based on complete ORF1 nucleotide sequences. Each branch is a representative sequence of a cluster, each cluster shows at least $20 \%$ nucleotide diversity with others. Clusters whose contain previously known complete ORF1 sequences are shaded grey, clusters with only sequences assembled in this study are shaded red. Scale is in number of substitutions per site. (c) Number of distinct anelloviruses of the same genus (i.e. Alpha-, Beta- and Gammatorquevirus) or in total co-detected in each positive sample. The horizontal lines in the box plots denote medians. Each dot represents one sample.

\section{Discussion}

The aim of this study was to provide a comprehensive characterization of the blood virome (i.e. viral landscape) in a population of young children with fever of unknown origin and/or severe outcomes who are living in Tanzania to pave the way of future clinical and diagnostic investigations. Beyond the well-recognized human viruses known to cause diseases and for some of them screened in routine clinical practice, or widely considered as "commensal," we identify numerous novel, divergent and unexpected viruses that show mNGS to be a powerful tool for the surveillance of unrecognized or emerging viral infections. Among these unexpected viruses, were a large proportion never before described in humans, of mammalian, arthropod or other invertebrate origin. As the detection of a viral sequence does not necessarily reflect the cause of the disease, further matched studies on a broader geographic, demographic and clinical scope would be required to better differentiate between background noise, abortive infections or novel causes of clinical disease.

Many previous investigations have surveyed viruses in the animal kingdom, including non-vertebrates, in order to extrapolate a probabilistic list of viruses that could emerge in humans [35-37]. However, this generates noise by reporting a vast majority of viruses which would not succeed in the stochastic process of a zoonotic transfer event or have not yet acquired the mutations with which to do. We propose that such surveillance should be complemented by large sentinel human virome populations that is much more likely to identify viral signatures of interest, that could guide future extended investigations.

We conducted two bioinformatics analyses in parallel for each sample: In addition to traditional de novo analysis, the raw data were analysed with the FeVir pipeline (specifically designed for this study and including the novel and particularly exhaustive Virosaurus reference database). The mNGS analysis reported the detection of a large diversity of RNA and DNA virus sequences. Of the 816 children, 394 (48.3\%) were found positive for at least one virus of "recognized clinical significance." Anelloviridae were detected in all except one sample and up to $92.2 \%$ of samples were found co-infected by all three anellovirus genera (TTV, TTMV and TTMDV). In addition, a large number of sequences were detected that related to 33 arthropod, vertebrate or mammalian virus species that are so far unknown to cause human infections; the most frequent being dicistroviruses, porcine parvoviruses 4-6 and ambidensoviruses.

To assess the presence of potential environmental or laboratory reagent contaminants, NTC and PC 


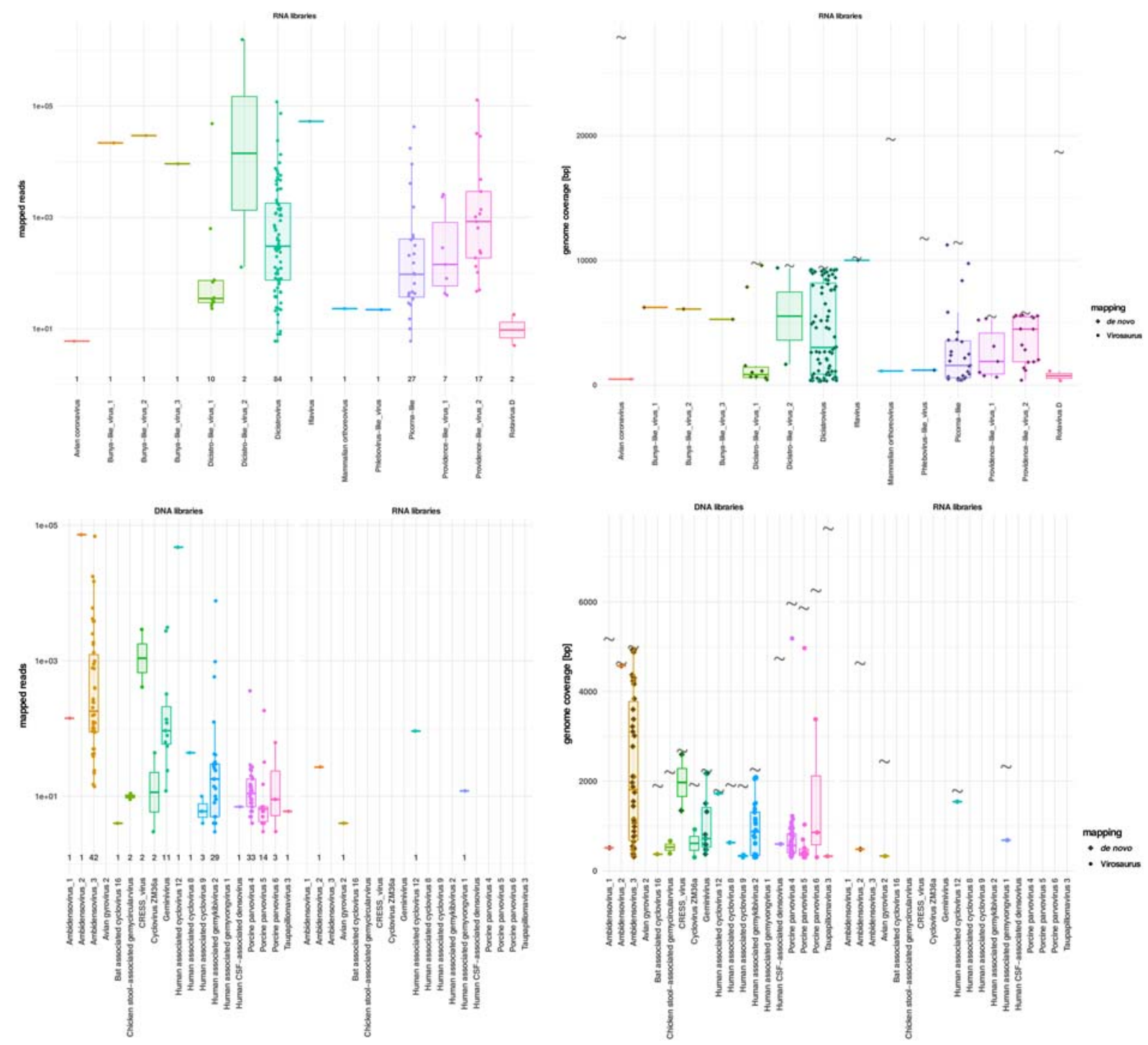

Figure 5. mNGS metrics for viruses of yet unknown significance. (a) Distribution of the mapped reads for RNA viruses. Each dot represents a positive sample. (b) RNA virus genome coverages. Each dot represents one sample. Viral full genome sizes (bp) are indicated by grey corrugated symbols (except for the segmented bunya-like virus 1-3). Viral sequences detected by de novo only are represented by grey diamonds. (c) Distribution of the mapped reads for DNA viruses detected in DNA (left side) and RNA (right side) libraries. Each dot represents a positive sample. (d) DNA virus genome coverages in DNA (left side) and RNA (right side) libraries. Each dot represents one sample. Viral full genome sizes (bp) are indicated by grey corrugated symbols. The horizontal lines in the box plots denote medians.

submitted to the whole procedure were included to each series. Despite this, mNGS data must be interpreted with caution (especially when the number of reads and/or positive samples is low) as sporadic contaminations cannot be ruled out e.g. the mNGS analysis reported in the blood for so-called respiratory viruses only one HPIV-4a positive sample with 4 reads detected as well as two human coronavirus OC43 positive samples with $\leq 10$ reads each (although they were collected in two different districts, Supplementary Figure 4) (Supplementary Table 1).

Concerning the viruses considered as being of "recognized clinical significance," sequences related to Herpesviridae, Picornaviridae and the Reoviridae family members were the most frequently detected among the 4 DNA (Herpesviridae > Adenoviridae > Parvoviridae > Polyomaviridae) and 9 RNA
(Picornaviridae $>$ Reoviridae $>$ Astroviridae, Caliciviridae $>$ Retroviridae $>$ Coronaviridae $>$ Flaviviridae $>$ Paramyxoviridae > Togaviridae) virus families observed (Supplementary Figure 1(a)). Indeed, among the 35 viral species detected and considered of clinical significance, we observed a predominance of human enteroviruses (13.8\%), rotaviruses (12\%), HHV- 6 (11\%) and HHV-7 (4.4\%). Overall, rotavirus A sequences were the most frequently detected and confirm that rotavirus infections frequently result in viremia in paediatric patients $[38,39]$. Similarly to HHV-6, HHV-7 was also frequently detected $(n=$ 36) in our cohort. Although commercial or in-house molecular assays are available, HHV-7 is rarely (if ever) tested even in high-resource settings. Although great care was taken during the RNA virus genome extraction (RNA protocol), we cannot rule out rare 
DNA contaminations from inter-/lower phases after phase separation. However, given their significant proportions, the detection of RNA for HHV-6 and HHV-7 in the RNA libraries suggest ongoing viral replication in the majority of positive cases. Some viruses are also notable for their absence, for instance, Epstein-Barr was not detected by mNGS analysis in any children. We also show the wide circulation of $\mathrm{EV}$ in East Africa, and report a potential new EVA71 genogroup $H$. Additional investigations on the prevalence and global distribution of this genogroup as well as its associated disease will give potentially important information about its neurotropic potential. Continuous genomic studies on the evolution of EV-A71 are important to detect new mutants or recombinants with epidemic potential [40]. Such surveillance is necessary, particularly as there is currently no treatment against this virus.

Interestingly, although its relationship with clinical pathology is currently difficult to establish, among different Picornaviridae family members we observed two samples harbouring Cardiovirus B. This virus was isolated in cell culture from stool samples collected from a child with fever of unknown origin and named the Saffold virus [41]. Since then, the virus has shown a wide circulation in many countries [42] and has been isolated from clinical specimens of children with gastrointestinal and respiratory symptoms as well as from cerebrospinal fluid samples of patients with aseptic meningitis. Recently, Ramesh and colleagues also reported the detection of Saffold virus sequences with mNGS in the serum of a febrile paediatric patient in Uganda [17]. Among the EV genera, one RV-C45/RV-C11 recombinant was observed. This recombinant was previously reported in human respiratory specimen collected in US [43] and our data suggest its circulation across Africa. Interestingly, nine of the $39 \mathrm{RV}-\mathrm{C}$ were detected by the de novo analysis only. This demonstrates that, although more time-consuming, a de novo approach is complementary to database-oriented pipelines and recommended in such surveillance studies to detect highly divergent viruses. To support this statement, $13 \mathrm{RV}-\mathrm{C}$ and $13 \mathrm{RV}-\mathrm{A}$ genomes were de novo assembled by Ramesh $\mathrm{A}$ and colleagues in their mNGS study [17].

Our results further highlight that HAstV has a significant circulation in the global population, with a higher prevalence of novel HAstV $(2.1 \%)$ than classical HAstV (0.9\%). This observation is in agreement with previous reports and underscores the clinical pertinence of novel HAstV in the paediatric population [44-46].

The potential clinical implication of Anelloviridae is still debated [47]. Among sera samples analysed in this study, 98.4\%, 97.4\% and 95.1\% were found positive for TTMV, TTV and TTMDV, respectively, which support a very early acquisition in life [48] with increased TTV and TTMDV prevalence in the paediatric population until 19-24 months of age before decreasing (modest age-related effect observed for TTMV) [49]. Anelloviridae are considered major members of the human blood virome and possibly one of the most abundant virus in blood samples [50]. Reflecting their genetic heterogeneity [51], anellovirus mixed populations are frequently observed in blood samples $[52,53]$. In agreement with these previous studies, we report a very high intra- and interindividual genetic heterogeneity for TTV, TTMV and TTMDV, with a co-detection rate of $99.0 \%$ (Figure 4). This is exemplified here by the finding that up to 69 different TTV, 259 TTMV and 189 TTMDV were observed in the serum from a single individual. Similar to $\mathrm{HPgV}-1$, TTV are known to interact with the immune system; yet the impact of these interactions is not well defined.

A wide range of RNA and DNA viruses previously unknown to infect humans were observed. These deserve specific attention in further studies in the febrile paediatric population in Africa. Indeed, the aim of this study was to characterize the diversity of the viral landscape in the blood of febrile Tanzanian children. However, while these viruses were discovered in febrile children, the absence of a non-febrile comparison group means that we cannot definitively associate the febrile episode to the presence of the viral sequences. While this specific question is beyond the scope of our study, the associations between the considerable diversity of metagenomics data and clinical features is yet to be explored. Therefore, although extremely challenging, it would be important to perform a similar mNGS survey analysis on blood samples from an asymptomatic paediatric cohort with comparable demographic and epidemiological characteristics to better clarify any potential clinical causality.

In conclusion, this blood virome study on a population of young children with fever of unknown origin and/or severe outcomes living in Tanzania highlighted several DNA and RNA viral sequences, and provides a large list of novel viruses that should be considered as a potential cause of human infections, some certainly being candidates for future fever investigations.

\section{Acknowledgements}

First, we would like to thank the patients and their caretakers for their participation and contribution to improved medical care for febrile illness in Tanzania. The authors would like to thank Lara Turin (University Hospitals of Geneva) and Brice Petit (University of Geneva) for technical assistance. S.C., M.A.H., J.F., V.D. and L.K. conceived and designed the study. F.L. performed bioinformatics and phylogenetic analyses. S.C., F.L., T.J., N.G., C.I., P.L.M., A.G., I.X. and L.K. developed and validated the bioinformatics 
pipeline “Fevir.” K.K., J.S., T.M., F.K., J.M., Z.S., H.T., and V.D. managed and performed sample collection. M.D. helped design and performed mNGS. G.V. performed sample extractions. G.E performed the spatio-temporal clusters of patients analysis. C.T. and MC.Z. contributed to the literature review. S.C., F.L. and MA.H. designed the figures. S.C., F.L., M.A.H. and L.K. wrote the manuscript. All authors revised and commented the manuscript. All authors read and approved the final version.

\section{Disclosure statement}

No potential conflict of interest was reported by the author(s).

\section{Funding}

This study was supported by the Bill and Melinda Gates Foundation [grant number OPP1163434] and by the Swiss National Science Foundation [grant number 320030_179507].

\section{Data availability}

The raw sequence data were deposited in the NCBI Sequence Read Archive under BioProject accession number PRJNA666535. Partial viral sequences $\geq$ 2000 nucleotides long obtained in this study were submitted to GenBank (accession no. MN727142MN727295 and MN765166-MN780477 for RNA and DNA viruses, respectively). The custom scripts that were used in this study are available at: https:// github.com/SAFIA-HTS/VIRAL-HTS.

\section{ORCID}

Samuel Cordey (D) http://orcid.org/0000-0002-2684-5680 Christian Iseli (D) http://orcid.org/0000-0002-2296-2863

\section{References}

[1] Williams SH, Cordey S, Bhuva N, et al. Investigation of the plasma virome from cases of unexplained febrile illness in Tanzania from 2013 to 2014: a comparative analysis between unbiased and VirCapSeq-VERT high-throughput sequencing approaches. mSphere. 2018;3(4):e00311-18.

[2] Zhang YZ, Chen YM, Wang W, et al. Expanding the RNA virosphere by unbiased metagenomics. Annu Rev Virol. 2019;6(1):119-139.

[3] Anthony SJ, Epstein JH, Murray KA, et al. A strategy to estimate unknown viral diversity in mammals. mBio. 2013;4(5):e00598-e00513.

[4] Rascovan N, Duraisamy R, Desnues C. Metagenomics and the human virome in asymptomatic individuals. Annu Rev Microbiol. 2016;70:125-141.

[5] Moustafa A, Xie C, Kirkness E, et al. The blood DNA virome in 8000 humans. PLoS Pathog. 2017;13(3): e1006292.

[6] Lau P, Cordey S, Brito F, et al. Metagenomics analysis of red blood cell and fresh-frozen plasma units. Transfusion. 2017;57(7):1787-1800.
[7] Li G, Zhou Z, Yao L, et al. Full annotation of serum virome in Chinese blood donors with elevated alanine aminotransferase levels. Transfusion. 2019;59 (10):3177-3185.

[8] Sauvage V, Laperche S, Cheval J, et al. Viral metagenomics applied to blood donors and recipients at high risk for blood-borne infections. Blood Transfus. 2016;14(5):400-407.

[9] Zhang W, Li L, Deng X, et al. Viral nucleic acids in human plasma pools. Transfusion. 2016;56(9):22482255.

[10] De Vlaminck I, Khush KK, Strehl C, et al. Temporal response of the human virome to immunosuppression and antiviral therapy. Cell. 2013;155(5):1178-1187.

[11] Schreiber PW, Kufner V, Hubel K, et al. Metagenomic virome sequencing in living donor-recipient kidney transplant pairs revealed JC polyomavirus transmission. Clin Infect Dis. 2018;69(6):987-994.

[12] Vu DL, Cordey S, Simonetta F. Human pegivirus persistence in human blood virome after allogeneic haematopoietic stem-cell transplantation. Clin Microbiol Infect. 2019;25(2):225-232.

[13] Li L, Deng X, Linsuwanon P, et al. AIDS alters the commensal plasma virome. J Virol. 2013;87 (19):10912-10915.

[14] Fahsbender E, Charlys da-Costa A, Elise Gill D, et al. Plasma virome of 781 Brazilians with unexplained symptoms of arbovirus infection include a novel parvovirus and densovirus. PLoS One. 2020;15(3): e0229993.

[15] Fernandes JF, Laubscher F, Held J, et al. Unbiased metagenomic next-generation sequencing of blood from hospitalized febrile children in Gabon. Emerg Microbes Infect. 2020;9(1):1242-1244.

[16] Ngoi CN, Siqueira J, Li L, et al. The plasma virome of febrile adult Kenyans shows frequent parvovirus B19 infections and a novel arbovirus (Kadipiro virus). J Gen Virol. 2016;97(12):3359-3367.

[17] Ramesh A, Nakielny S, Hsu J, et al. Metagenomic nextgeneration sequencing of samples from pediatric febrile illness in Tororo, Uganda. PLoS One. 2019;14(6): e0218318.

[18] Wylie KM, Mihindukulasuriya KA, Sodergren E, et al. Sequence analysis of the human virome in febrile and afebrile children. PLoS One. 2012;7(6):e27735.

[19] Maze MJ, Bassat Q, Feasey NA, et al. The epidemiology of febrile illness in sub-Saharan Africa: implications for diagnosis and management. Clin Microbiol Infect. 2018;24(8):808-814.

[20] Keitel K, Kagoro F, Samaka J, et al. A novel electronic algorithm using host biomarker point-of-care tests for the management of febrile illnesses in Tanzanian children (e-POCT): a randomized, controlled noninferiority trial. PLoS Med. 2017;14(10):e1002411.

[21] Gleizes A, Laubscher F, Guex S, et al. Virosaurus a reference to explore and capture virus genetic diversity. Viruses. 2020;12(11):1248.

[22] Brito F, Manni M, Laubscher F, et al. Novel Rhabdovirus and an almost complete drain fly transcriptome recovered from two independent contaminations in clinical samples. bioRxiv. 2019. doi:10. $1101 / 645325$.

[23] Petty T, Cordey S, Padioleau I, et al. Comprehensive human virus screening using high-throughput sequencing with a user-friendly representation of bioinformatics analysis: a pilot study. J Clin Microbiol. 2014;52(9):3351-3361. 
[24] Virusscan: https://github.com/sib-swiss/virusscan.

[25] Junier $T$, Huber $M$, Schmutz $S$, et al. Viral Metagenomics in the clinical realm: lessons learned from a Swiss-wide ring trial. Genes. 2019;10(9):655.

[26] Asplund M, Kjartansdottir KR, Mollerup S, et al. Contaminating viral sequences in high-throughput sequencing viromics: a linkage study of 700 sequencing libraries. Clin Microbiol Infect. 2019;25 (10):1277-1285.

[27] Lipkin WI. The changing face of pathogen discovery and surveillance. Nat Rev Microbiol. 2013;11 (2):133-141.

[28] Laubscher F, Cordey S, Hartley MA, et al. Nearly complete genome sequence of a novel phlebovirus-like virus detected in a human plasma sample by highthroughput sequencing. Microbiol Resour Announc. 2019;8(35):e00764-19.

[29] Zaharia M, Bolosky W, Curtis K, et al. Faster and more accurate sequence alignment with SNAP. arXiv 2011;111.5572.

[30] Peng Y, Leung HC, Yiu SM, et al. IDBA-UD: a de novo assembler for single-cell and metagenomic sequencing data with highly uneven depth. Bioinformatics. 2012;28(11):1420-1428.

[31] Camacho C, Coulouris G, Avagyan V, et al. BLAST+: architecture and applications. BMC Bioinform. 2009;10:421.

[32] Database U-Rv: https://rvdb-prot.pasteur.fr/.

[33] Scully EJ, Basnet S, Wrangham RW, et al. Lethal respiratory disease associated with human rhinovirus $C$ in wild chimpanzees, Uganda, 2013. Emerg Infect Dis. 2018;24(2):267-274.

[34] Cordey S, Laubscher F, Hartley MA, et al. Detection of dicistroviruses RNA in blood of febrile Tanzanian children. Emerg Microbes Infect. 2019;8 (1):613-623.

[35] Chandler JA, Liu RM, Bennett SN. RNA shotgun metagenomic sequencing of northern California (USA) mosquitoes uncovers viruses, bacteria, and fungi. Front Microbiol. 2015;6:185.

[36] Delwart E. Animal virus discovery: improving animal health, understanding zoonoses, and opportunities for vaccine development. Curr Opin Virol. 2012;2 (3):344-352.

[37] Sadeghi M, Altan E, Deng X, et al. Virome of $>12$ thousand Culex mosquitoes from throughout California. Virology. 2018;523:74-88.

[38] Blutt SE, Matson DO, Crawford SE, et al. Rotavirus antigenemia in children is associated with viremia. PLoS Med. 2007;4(4):e121.

[39] Chitambar SD, Tatte VS, Dhongde R, et al. High frequency of rotavirus viremia in children with acute gastroenteritis: discordance of strains detected in stool and sera. J Med Virol. 2008;80(12):2169-2176.
[40] Yip CC, Lau SK, Woo PC, et al. Human enterovirus 71 epidemics: what's next? Emerg Health Threats J. 2013;6:19780.

[41] Jones MS, Lukashov VV, Ganac RD, et al. Discovery of a novel human picornavirus in a stool sample from a pediatric patient presenting with fever of unknown origin. J Clin Microbiol. 2007;45(7):2144-2150.

[42] Tan SZ, Tan MZ, Prabakaran M. Saffold virus, an emerging human cardiovirus. Rev Med Virol. 2017;27(1):e1908.

[43] Liggett SB, Bochkov YA, Pappas T, et al. Genome sequences of rhinovirus $\mathrm{C}$ isolates from wisconsin pediatric respiratory studies. Genome Announc. 2014;2(2):e00203-14.

[44] Cordey S, Vu DL, Zanella MC, et al. Novel and classical human astroviruses in stool and cerebrospinal fluid: comprehensive screening in a tertiary care hospital, Switzerland. Emerg Microbes Infect. 2017;6(9): e84.

[45] Khamrin P, Thongprachum A, Okitsu S, et al. Multiple astrovirus MLB1, MLB2, VA2 clades, and classic human astrovirus in children with acute gastroenteritis in Japan. J Med Virol. 2016;88(2):356-360.

[46] Vu DL, Sabrià A, Aregall N, et al. Novel human astroviruses: prevalence and association with common enteric viruses in undiagnosed gastroenteritis cases in Spain. Viruses. 2019;11(7):585.

[47] Spandole S, Cimponeriu D, Berca LM, et al. Human anelloviruses: an update of molecular, epidemiological and clinical aspects. Arch Virol. 2015;160(4):893-908.

[48] Maggi F, Bendinelli M. Human anelloviruses and the central nervous system. Rev Med Virol. 2010;20 (6):392-407.

[49] McElvania TeKippe E, Wylie KM, Deych E, et al. Increased prevalence of anellovirus in pediatric patients with fever. PLoS One. 2012;7(11):e50937.

[50] Zanella MC, Cordey S, Kaiser L. Beyond cytomegalovirus and Epstein-Barr virus: a review of viruses composing the blood virome of solid organ transplant and hematopoietic stem cell transplant recipients. Clin Microbiol Rev. 2020;33(4):e00027-20.

[51] Biagini P. Classification of TTV and related viruses (anelloviruses). Curr Top Microbiol Immunol. 2009;331:21-33.

[52] Eibach D, Hogan B, Sarpong N, et al. Viral metagenomics revealed novel betatorquevirus species in pediatric inpatients with encephalitis/meningoencephalitis from Ghana. Sci Rep. 2019;9(1):2360.

[53] Ninomiya $M$, Takahashi $M$, Nishizawa $T$, et al. Development of PCR assays with nested primers specific for differential detection of three human anelloviruses and early acquisition of dual or triple infection during infancy. J Clin Microbiol. 2008;46 (2):507-514. 